ISSN 1991-8631

Original Paper

http://indexmedicus.afro.who.int

\title{
Libération comparée de sels dans l'eau par la minéralisation de l'azolla, la bouse de vache, la fiente de volaille et les sons de riz et de maïs utilisés en pisciculture
}

\author{
Hyppolite AGADJIHOUEDE ${ }^{1 *}$, Elie MONTCHOWUI ${ }^{1,2}$, Antoine CHIKOU ${ }^{1}$ et \\ Philippe A. LALEYE ${ }^{1}$ \\ ${ }^{1}$ Laboratoire d'Hydrobiologie et d'Aquaculture, Faculté des Sciences Agronomiques, Université d'Abomey- \\ Calavi, 01 BP 526 Cotonou, Bénin. \\ ${ }^{2}$ Ecole National Supérieure des Sciences et Techniques Agronomiques de Kétou, BP95 Kétou, Bénin. \\ "Auteur correspondant, E-mail : agadjihouede@yahoo.fr
}

\section{RESUME}

Dans le but d'identifier le meilleur fertilisant pour la production du zooplancton, un essai de minéralisation de 5 substrats (azolla, bouse de vache, fiente de volaille, son de riz et son de maïs) et de libération comparée de sels dans l'eau a été réalisé. L'expérimentation est conduite dans 10 bacs contenant chacun 15 litres d'eau. Dans chaque bac est plongé $50 \mathrm{~g}$ de substrat sec emballé dans une toile moustiquaire à fine maille. Toutes les 72 heures, les paramètres de minéralisation tels que le rapport $\mathrm{C} / \mathrm{N}$ résiduels dans les substrats, le TDS, la conductivité, la salinité de l'eau et le taux des sels nutritifs dissous sont déterminés. Après18 jours, les rapports $\mathrm{C} / \mathrm{N}$ moyens, les plus faibles, sont respectivement de 7,80 $\pm 0,31 ; 11,05 \pm 0,8$ et $11,32 \pm 0,3$ pour la fiente de volaille, la bouse de vache et l'azolla. Ces rapports, très élevés, sont respectivement de 32,27 \pm 4,58 et 35,77 $\pm 5,46$ pour les sons de maïs et du riz. Les taux de TDS, de conductivité, de la salinité et des sels dissous ont accru. La fiente de volaille présente les plus forts taux de TDS, de conductivité, de salinité, de $\mathrm{N}-\mathrm{NH}_{4}, \mathrm{~N}-\mathrm{NO}_{3}, \mathrm{~N}$ inorganique et $\mathrm{P}_{-} \mathrm{PO}_{4}$. Elle est suivie de la bouse de vache, de l'azolla, du son de maïs et du son de riz. L'analyse hiérarchique ascendante et l'analyse en composante principale range les substrats en 3 groupes. Le groupe I est composé de la fiente de volaille, le groupe II de la bouse de vache et de l'azolla et le groupe III des sons de maïs et du riz. La fiente de volaille et la bouse de vache sont les meilleurs substrats.

(C) 2011 International Formulae Group. All rights reserved.

Mots clés : Substrats, minéralisation, libération, sels dissous, pisciculture.

\section{INTRODUCTION}

Dans le but de valoriser les sous produits agricoles en aquaculture, certains sous produits sont de plus en plus utilisés comme fertilisant des étangs. Pour ce faire, en pisciculture rurale, dans les pays africains, les sons de riz et de maïs, les déjections de volaille, de vache et du porc sont les plus utilisés (Dabbadie, 1996; Awaïss et Kestemont, 1997 ; Prithwiraj et al., 2004 ; Dossou, 2008).

De nombreuses études ont décrit les relations trophiques reliant les organismes de la chaîne trophique classique (nutriments- 
phytoplancton - zooplancton - poisson) (Fenchel, 1987 ; Porter et al., 1988). Il a été admis que l'ajout de nutriments accroît la biomasse du phytoplancton, et celle du zooplancton (Dillon et Rigler, 1974 ; Dabbadié, 1996; Burns et Schallenberg, 1998 ; Agadjihouèdé et al., 2010).

Tout récemment, la fougère aquatique (Azolla filiculoides) a été utilisée avec succès dans la pisciculture pour améliorer les conditions trophiques des étangs et par conséquent augmenter la production piscicole avec un apport relativement faible d'intrant et de fertilisants minéraux (Abou, 2007).

L'efficacité de ces sous produits dans la production du zooplancton réside dans leur aptitude à libérer les sels nutritifs indispensables dans l'eau pour la production primaire. Ces nutriments sont surtout les composés azotés et phosphatés. Selon Rigler et Peters (1995), l'azote et le phosphore constituent les principaux facteurs limitants pour la production de zooplancton dans les écosystèmes aquatiques. On a souvent étudié les effets des nutriments sur la production du phytoplancton et du zooplancton, mais à notre connaissance, très peu font le lien entre le fertilisant et la quantité de nutriments libérés qui peuvent pourtant orienter dans le choix du fertilisant.

Le but de cette étude est de déterminer la quantité et la vitesse de libération de chacun de ces sous-produits afin de comprendre lequel convient le mieux.

\section{MATERIEL ET METHODES \\ Protocole expérimental}

Le test de minéralisation et de libération des sels dissous est réalisé dans 10 bacs plastiques circulaires de 20 litres de contenance (diamètre $=35 \mathrm{~cm}$, hauteur $=18$ $\mathrm{cm})$. Ces bacs sont remplis avec 15 litres d'eau de robinet et sont disposés dans des bassins couverts de branchages pour diminuer l'incidence des rayons solaires. Les 5 substrats (azolla, bouse de vache, fiente de volaille, son de riz et son de maïs) sont répartis en répliquas soit au total 10 bacs. Dans chaque bac, $50 \mathrm{~g}$ de substrat sec sont introduits. L'expérimentation a durée 18 jours. La fréquence de mesure est de 72 heures.

\section{Minéralisation des substrats}

A des fins de fertilisation, la minéralisation du substrat est un critère important de qualité (Sérémé, 2007). Elle est évaluée à travers l'évolution du rapport $\mathrm{C} / \mathrm{N}$ résiduels dans le substrat, de la valeur des solides totaux dissous (TDS), de la conductivité et de la salinité de l'eau fertilisée. A cet effet, toutes les 72 heures, nous avons mesuré in situ le TDS, la conductivité et la salinité au moyen d'un conductimètre de type WTW-LF 340. Nous avons déterminé la quantité d'azote organique total $(\mathrm{N})$ par la méthode de Kjedahl et celle de résidu du carbone organique total (C) par la méthode de Walkley-Black (Bunasols, 1987; Baize, 1988). Le rapport de minéralisation $\mathrm{C} / \mathrm{N}$ des substrats est calculé.

\section{Dosage des sels nutritifs}

Les sels nutritifs libérés dans les bacs sont également dosés toutes les 72 heures après l'installation de l'essai. Pour ce faire, un échantillon de $100 \mathrm{ml}$ d'eau est prélevé dans chaque bac. L'azote ammoniacal $\left(\mathrm{N}-\mathrm{NH}_{4}\right)$, l'azote nitrique $\left(\mathrm{N}-\mathrm{NO}_{3}\right)$, l'azote nitreux $(\mathrm{N}-$ $\left.\mathrm{NO}_{2}\right)$ et l'(ortho)phosphate $\left(\mathrm{P}-\mathrm{PO}_{4}\right)$ sont mesurés à l'aide du spectrophotomètre $\mathrm{HACH}$ (DR/2010) (Hach, 1991; Greenberg et al.,1992).

Le nitrate a été dosé par la méthode de réduction au cadmium. En effet, les ions nitrates sont réduits en ions nitrites qui réagissent en milieu acide avec l'acide sulfanilique pour former un sel diazonium intermédiaire. Ce sel se lie avec l'acide chromotropique pour former un composé de 
couleur rose. La densité optique est mesurée à 500 nm (Hach, 1991; Greenberg et al., 1992). Le nitrite a été dosé par la méthode de diazotation. C'est la procédure finale de dosage du nitrate. Les ions nitrites réagissent en milieu acide pour former un sel de diazonium qui se combine ensuite à l'acide chromotropique pour former un composé coloré. La densité optique est mesurée à 507 nm (Hach, 1991 ; Greenberg et al., 1992).

Pour l'azote ammoniacal, la méthode de Nessler a été utilisée. Les ions ammonium réagissent avec le réactif de Nessler $(\mathrm{HgI} 2$ et $\mathrm{KI}$ en solution dans de la soude) pour former une coloration jaune. Une solution stabilisante (à base d'EDTA) est utilisée pour éviter la précipitation du calcium ou du magnésium lors de l'addition du réactif de Nessler. La densité optique est mesurée à 425 nm (Hach, 1991 ; Greenberg et al., 1992).

Quant au phosphate, il a été déterminé par la méthode de l'acide ascorbique. L'orthophosphate réagit avec le molybdate en milieu acide pour former un complexe phosphomolybdate. L'acide ascorbique réduit ensuite ce complexe, ce qui produit une couleur bleue. La densité optique est mesurée à 890 nm (Hach, 1991 ; Greenberg et al., 1992).

\section{Analyse statistique}

Le test de Hartley (Hartley, 1959) a été utilisé pour tester l'homogénéité des variables issues du dosage des paramètres nutritifs des bacs expérimentaux. Ensuite, les comparaisons ont été faite en utilisant l'analyse non-paramétrique de Mann-Whitney (Jobling et al., 1994). Enfin, l'analyse en composantes principales (ACP) a été employée pour dégager d'éventuelles corrélations entre la distribution des substrats et les différentes variables nutritives $\left(\mathrm{N}-\mathrm{NH}_{4}\right.$, $\mathrm{N}-\mathrm{NO}_{3}, \mathrm{~N}-\mathrm{NO}_{2}, \mathrm{~N}$ inorganique, $\mathrm{P}-\mathrm{PO}_{4}$ ) et les variables de minéralisation des substrats
(TDS, conductivité, salinité, $\mathrm{C} / \mathrm{N}$ ) afin de regrouper les substrats semblables.

Toutes ces analyses ont été effectuées à l'aide du logiciel STATISTICA 6.1.

\section{RESULTATS}

Les Tableaux 1 et 2 présentent respectivement la composition chimique des 5 substrats et la synthèse des résultats de minéralisation de ces substrats.

\section{Minéralisation des différents substrats Variation de C/N résiduels des substrats}

Le taux de $\mathrm{C} / \mathrm{N}$ a décru dans tous les bacs du début jusqu'à la fin de l'expérimentation (Figure 1). Dans les bacs à azolla (AZ), à bouse (BZ) et à fiente (FV), la décroissance de $\mathrm{C} / \mathrm{N}$ est très faible en passant de $8,2 \pm 0,12$ (début) à 7,39 $\pm 0,08$ (fin) pour $\mathrm{AZ}$, de 11,69 $\pm 0,32$ (début) à 9,45 $\pm 0,21$ (fin) pour la BV et de 11,52 $\pm 0,1$ (début) à $10,7 \pm 0,05$ (fin) pour la FV. La variation n'est pas significative entre les valeurs du début et de la fin ( $p>0,05)$. Mais dans les milieux à sons (SM et SR), la décroissance est significative $(\mathrm{p}<0,05)$. Les valeurs les plus faibles du rapport $\mathrm{C} / \mathrm{N}$ sont enregistrées dans les bacs à azolla et les plus fortes dans les bacs à SR. Il n'existe aucune différence significative de $\mathrm{C} / \mathrm{N}$ entre les bacs à azolla, à bouse et à fiente $(\mathrm{p}>0,05)$. Mais la différence est significative entre le bac à sons de maïs et celui à son de riz qui ne sont pas significativement différents entre eux.

\section{Variations de TDS, conductivité et de salinité dans les bacs}

Les taux de TDS ont significativement augmenté dans tous les bacs du début jusqu'à la fin de l'expérimentation $(\mathrm{p}<0,05)$ (Figure 2). En 18 jours, ces taux de solides dissous sont passés de $30,21 \pm 0,33 \mathrm{mg} / \mathrm{g}$ dans tous les bacs à 171,75 mg/g, 237,28 mg/g, 223 mg/g, $140,40 \mathrm{mg} / \mathrm{g}$ et $129 \mathrm{mg} / \mathrm{g}$ respectivement dans les bacs à $\mathrm{AZ}, \mathrm{BV}, \mathrm{FV}, \mathrm{SM}$ et à $\mathrm{SR}$. Les valeurs moyennes de TDS obtenues sont plus 
élevées $(160,65 \pm 14,20$ mg/g) dans les bacs à FV. Elles sont suivies respectivement de celles des bacs à BV $(137,22 \pm 13,29 \mathrm{mg} / \mathrm{g})$, AZ $(98,30 \pm 16,95 \mathrm{mg} / \mathrm{g}), \mathrm{SM}(86,13 \pm 13,08$ $\mathrm{mg} / \mathrm{g})$ et à SR $(79,21 \pm 11,91 \mathrm{mg} / \mathrm{g})$. Dans les bacs à FV, le TDS ne varie pas significativement $(p>0,05)$. Mais, il est significativement différent des valeurs de TDS obtenues dans les bacs à $\mathrm{AZ}$ et à sons (SM et SR) $(\mathrm{p}<0,05)$. Dans ces bacs à sons, il n'existe aucune différence significative entre les valeurs de TDS ( $p>0,05)$.

Du début à la fin de l'expérimentation, les valeurs de conductivité et de salinité tout comme celles du TDS, ont significativement cru dans tous les bacs $(\mathrm{p}<0,05)$ à l'exception des bacs à sons (SM et SR) où l'augmentation a commencé à partir du $6^{\text {ème }}$ jour (Figures 3 et 4). Mais, les valeurs moyennes de ces deux paramètres ne varient pas significativement entre les bacs $(p>0,05)$ sauf dans les bacs à $\mathrm{FV}$ et à sons (SM et $\mathrm{SR})$ où la différence est significative $(\mathrm{p}<0,05)$.

\section{Qualité nutritive de l'eau}

Elle est évaluée à travers les taux de sels nutritifs (N-NH4, N-NO3, N-NO2, N inorganique total, P-PO4) libérés dans les bacs.

\section{Variations des sels minéraux libérés dans les bacs}

L'évolution des valeurs des sels nutritifs dissous est représentée à la Figure 5. Les concentrations de $\mathrm{N}-\mathrm{NH}_{4}, \mathrm{~N}-\mathrm{NO}_{3}, \mathrm{~N}-\mathrm{NO}_{2}$, $\mathrm{N}$ inorganique total et le $\mathrm{P}-\mathrm{PO} 4$ qui étaient respectivement de $0,11 \mathrm{mg} / \mathrm{L}, 0,1 \mathrm{mg} / \mathrm{L}, 0,0$ $\mathrm{mg} / \mathrm{L}, 0,21 \mathrm{mg} / \mathrm{L}$ et $11,9 \mathrm{mg} / \mathrm{L}$ au début de l'expérimentation. Au bout des 18 jours dans tous les bacs, la concentration de N-NH4, a atteint 13,65 $\mathrm{mg}$ (moyenne $=11,58 \pm 5,43$ $\mathrm{mg} / \mathrm{L})$ dans les bacs à $\mathrm{AZ}, 19,50 \mathrm{mg} / \mathrm{L}(13,39$ $\pm 07,1 \mathrm{mg} / \mathrm{L})$ dans les bacs à $\mathrm{BV}, 39 \mathrm{mg} / \mathrm{L}$ $(32,40 \pm 14,42 \mathrm{mg} / \mathrm{L})$ dans les bacs à $\mathrm{FV}$, $13,65 \mathrm{mg} / \mathrm{L}(5,41 \pm 4,61 \mathrm{mg} / \mathrm{L})$ dans les bacs à $\mathrm{SM}$ et $0,78 \mathrm{mg} / \mathrm{L}(0,41 \pm 0,2 \mathrm{mg} / \mathrm{L})$ dans les bacs à SR.

Les quantités de N-NO3 dosées ont varié entre 0,1 et $15 \mathrm{mg} / \mathrm{L}$ dans les bacs à $\mathrm{AZ}$ et à $\mathrm{BV}$ avec des moyennes respectives de $7,87 \pm 6,34 \mathrm{mg} / \mathrm{L}$ et de 10,90 $\pm 5,67 \mathrm{mg} / \mathrm{L}$. Elles ont atteint des valeurs plus importantes dans les bacs à $\mathrm{FV}$ avec une moyenne de $20,73 \pm 15,37 \mathrm{mg} / \mathrm{L}$. Dans les bacs à $\mathrm{SM}$ et à $\mathrm{SR}$, la quantité de $\mathrm{N}-\mathrm{NO}_{3}$ dosée est restée faible. Les valeurs enregistrées varient entre 0,1 et $7,5 \mathrm{mg} / \mathrm{L}(2,76 \pm 3,33 \mathrm{mg} / \mathrm{L})$ pour le SM et entre 0,1 et $1,1 \mathrm{mg} / \mathrm{L}(0,6 \pm 0,36 \mathrm{mg} / \mathrm{L})$ pour le SR.

Le taux de N-NO2 est faible dans tous les bacs et reste inférieur à $0,3 \mathrm{mg} / \mathrm{L}$ à l'exception des bacs à bouse où il a atteint $1,13 \mathrm{mg} / \mathrm{L}$. L'azote inorganique calculé varie entre 0,21 et $28,8 \mathrm{mg} / \mathrm{L}(19,51 \pm 10,0 \mathrm{mg} / \mathrm{L})$ dans les bacs à $A Z$, entre 0,21 et $34,61 \mathrm{mg} / \mathrm{L}$ $(23,91 \pm 12,65 \mathrm{mg} / \mathrm{L})$ dans les bacs à $\mathrm{BV}$, entre 0,21 et $89,15 \mathrm{mg} / \mathrm{L} \quad(53,29 \pm 27,00$ $\mathrm{mg} / \mathrm{L}$ ) dans les bacs à $\mathrm{FV}$, entre 0,21 et 15,32 $\mathrm{mg} / \mathrm{L}(8,26 \pm 6,38 \mathrm{mg} / \mathrm{L})$ dans les bacs à $\mathrm{SM}$ et entre 0,21 et $1,59 \mathrm{mg} / \mathrm{L}(1,00 \pm 0,50 \mathrm{mg} / \mathrm{L})$ dans les bacs à SR.

Les taux de $\mathrm{P}_{-} \mathrm{PO}_{4}$ libérés dans l'eau sont élevés et atteignent $144 \mathrm{mg} / \mathrm{L}$ dans les bacs à $\mathrm{BV}$ et à $\mathrm{FV}$ avec des moyennes respectives de 69,58 $\pm 50,26 \mathrm{mg} / \mathrm{L}$ et de 92,04 $\pm 60,15 \mathrm{mg} / \mathrm{L}$. Dans les bacs à $\mathrm{AZ}, \mathrm{SM}$ et à $\mathrm{SR}$, les valeurs maximales et moyennes enregistrées sont respectivement $84 \mathrm{mg} / \mathrm{L}$ $(49,53 \pm 33,73 \mathrm{mg} / \mathrm{L}), 96 \mathrm{mg} / \mathrm{L}(54,74 \pm 45,16$ $\mathrm{mg} / \mathrm{L})$ et $3,6 \mathrm{mg} / \mathrm{L}(1,91 \pm 1,28 \mathrm{mg} / \mathrm{L})$.

Le test de Mann-Whitney montre que les meilleurs taux de $\mathrm{N}-\mathrm{NH}_{4}, \mathrm{~N}-\mathrm{NO}_{3}$ et $\mathrm{N}$ inorganique sont enregistrés dans les bacs à $\mathrm{FV}(\mathrm{p}<0,05)$. Les taux de $\mathrm{N}-\mathrm{NH}_{4}, \mathrm{~N}-\mathrm{NO}_{3}$ et $\mathrm{N}$ inorganique ne varient pas significativement dans les bacs à $\mathrm{AZ}$ et à $\mathrm{BV}$ ( $\mathrm{p}>0,05$ ); mais ils sont significativement différents $(\mathrm{p}<0,05)$ des taux enregistrés dans les bacs à SM et à SR où la différence n'est pas significative $(p>0,05)$. Cependant, les 
taux de $\mathrm{N}_{-} \mathrm{NO}_{2}$ ne varient pas significativement dans tous les bacs $(p>0,05)$ à l'exception des bacs à $\mathrm{BV}$. Pour le $\mathrm{P}-\mathrm{PO}_{4}$, il n'existe aucune différence significative entre tous les bacs $(\mathrm{p}>0,05)$ à l'exception des bacs à $\mathrm{SR}$ où les valeurs enregistrées sont plus faibles $(\mathrm{p}<0,05)$.

\section{Types de substrats et les variables chimiques}

La classification hiérarchique ascendante (AHC) et l'analyse en composante principale appliquées à la matrice substrats/caractéristiques (5 substrats et 9 variables chimiques), ont permis de distinguer trois groupes de substrats/variables chimiques (Figure 6). Pour l'ensemble des variables chimiques, l'ordination des corrélations par l'analyse en composante principale (ACP) indique que l'axe I (valeur propre $\lambda 1=6,54$ ) et l'axe II (valeur propre $\lambda 2=0,85$ ) expriment $92,39 \%$ de la variance cumulée. Le groupe I (FV1 et FV2) est constitué uniquement de la fiente de volaille. Ce groupe est corrélé négativement à l'axe I et aux variables environnementales que sont conductivité, salinité, $\mathrm{N}-\mathrm{NH}_{4}$ et $\mathrm{N}-\mathrm{NO}_{3}$ de l'eau. Le groupe II (AZ1, AZ2, BV1 et BV2), fortement corrélé à l'axe II, correspond à 2 substrats caractérisés surtout par le $\mathrm{N}^{-} \mathrm{NO}_{2}$. Il comprend l'azolla et la bouse de vache. Le groupe III (SM1, SM2, SR1 et SR2) est fortement corrélé à l'axe I. Il décrit aussi 2 substrats que sont les sons de maïs et de riz caractérisés par le rapport $\mathrm{C} / \mathrm{N}$.

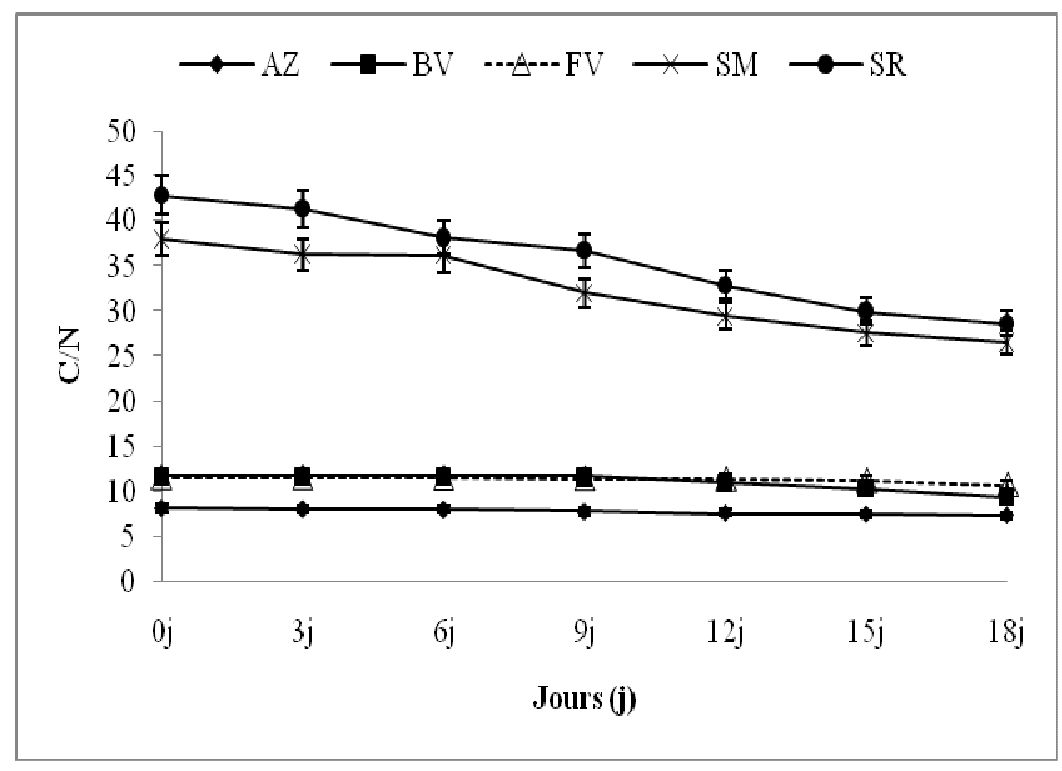

Figure 1 : Evolution du rapport $\mathrm{C} / \mathrm{N}$ résiduel dans les bacs au cours de l'expérience. $\mathrm{AZ}=$ Azolla, $\mathrm{BV}=$ Bouse de vache, $\mathrm{FV}=$ Fiente de volaille, $\mathrm{SM}=$ Son de maïs, $\mathrm{SR}=$ Son de riz . 


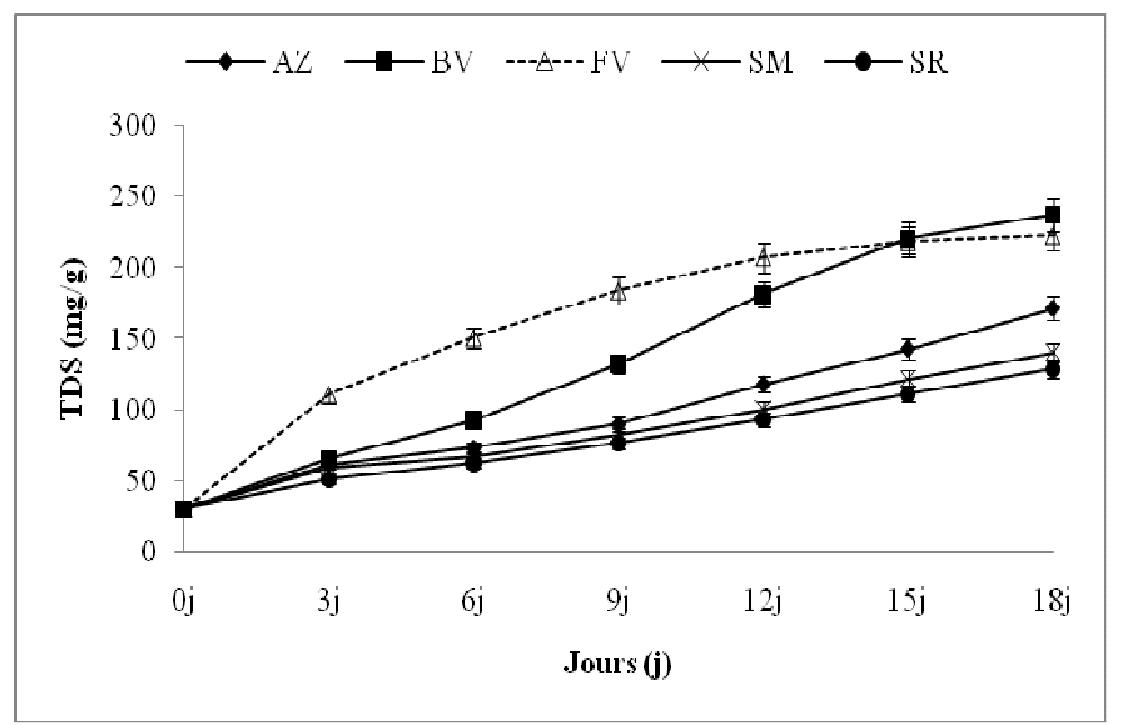

Figure 2 : Evolution du TDS dans les bacs au cours de l'expérience.

$\mathrm{AZ}=$ Azolla, $\mathrm{BV}=$ Bouse de vache, $\mathrm{FV}=$ Fiente de volaille, $\mathrm{SM}=$ Son de maïs, $\mathrm{SR}=$ Son de riz .

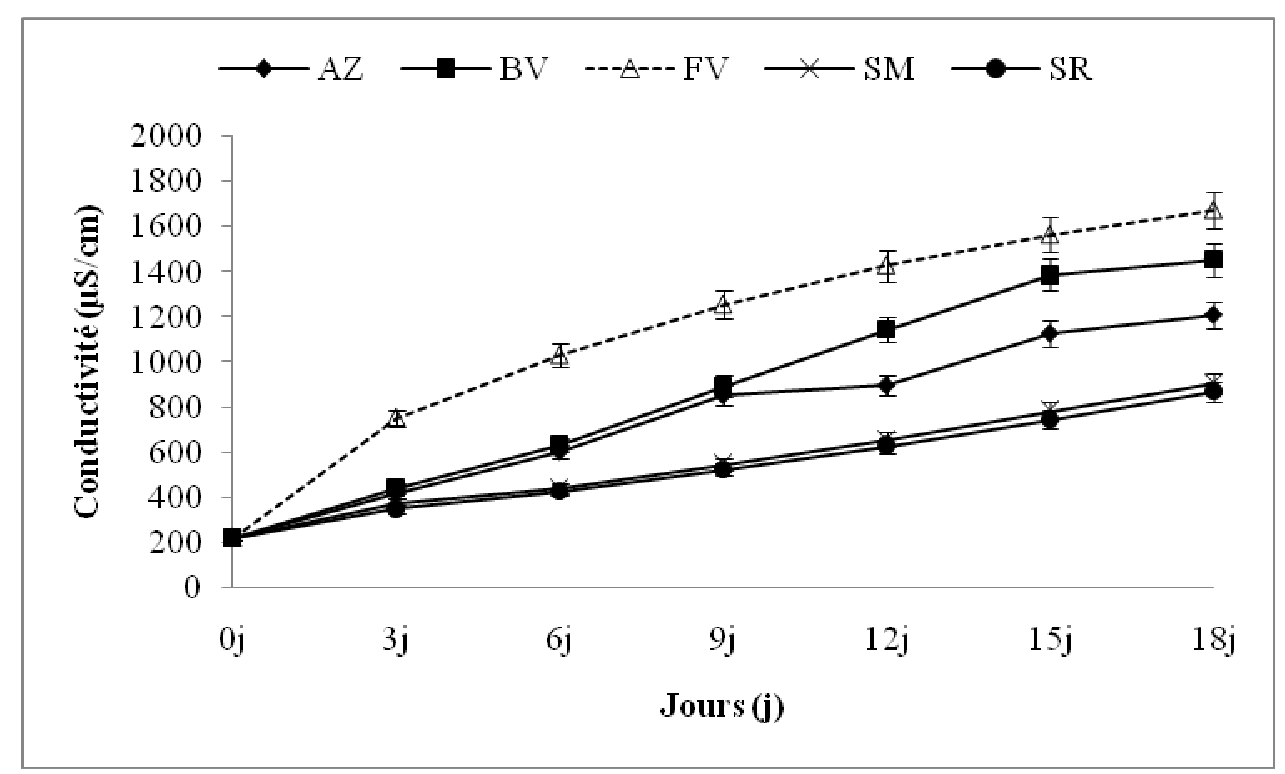

Figure 3 : Evolution de la conductivité dans les bacs au cours de l'expérience. $\mathrm{AZ}=$ Azolla, $\mathrm{BV}=$ Bouse de vache, $\mathrm{FV}=$ Fiente de volaille, $\mathrm{SM}=$ Son de maiis, $\mathrm{SR}=$ Son de riz . 


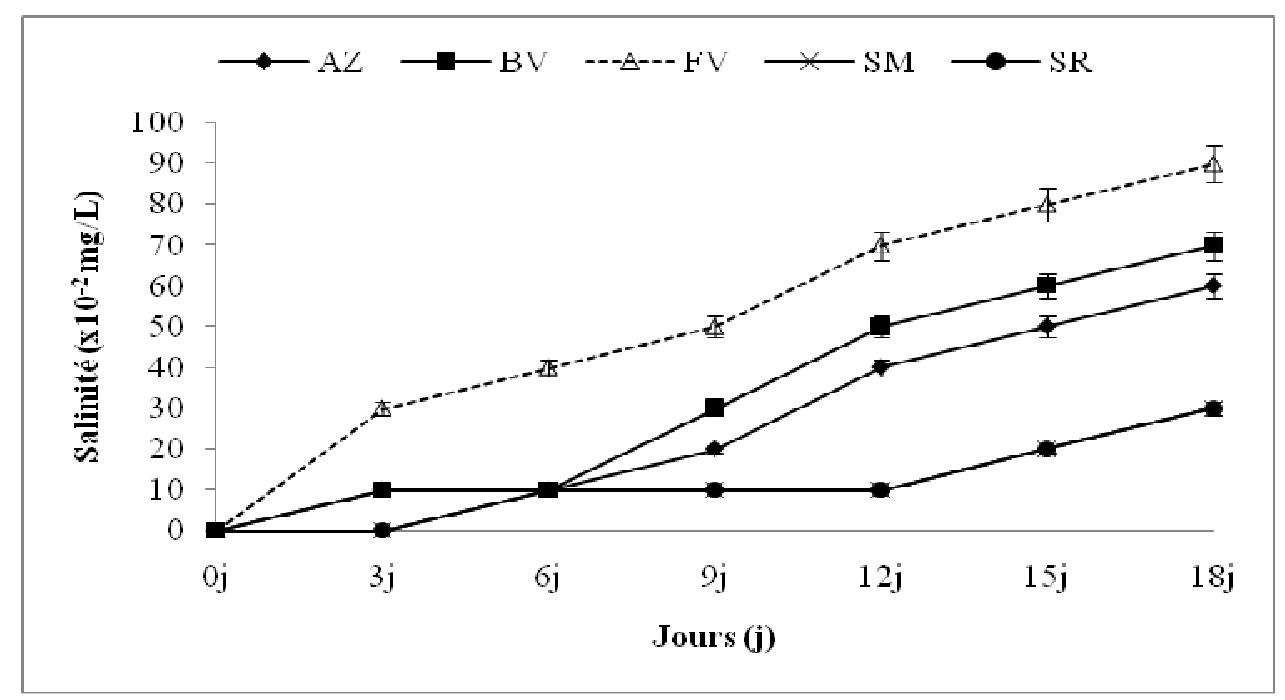

Figure 4 : Evolution de la salinité dans les bacs au cours de l'expérience.

$\mathrm{AZ}=$ Azolla, $\mathrm{BV}=$ Bouse de vache, $\mathrm{FV}=$ Fiente de volaille, $\mathrm{SM}=$ Son de maïs, $\mathrm{SR}=$ Son de riz.
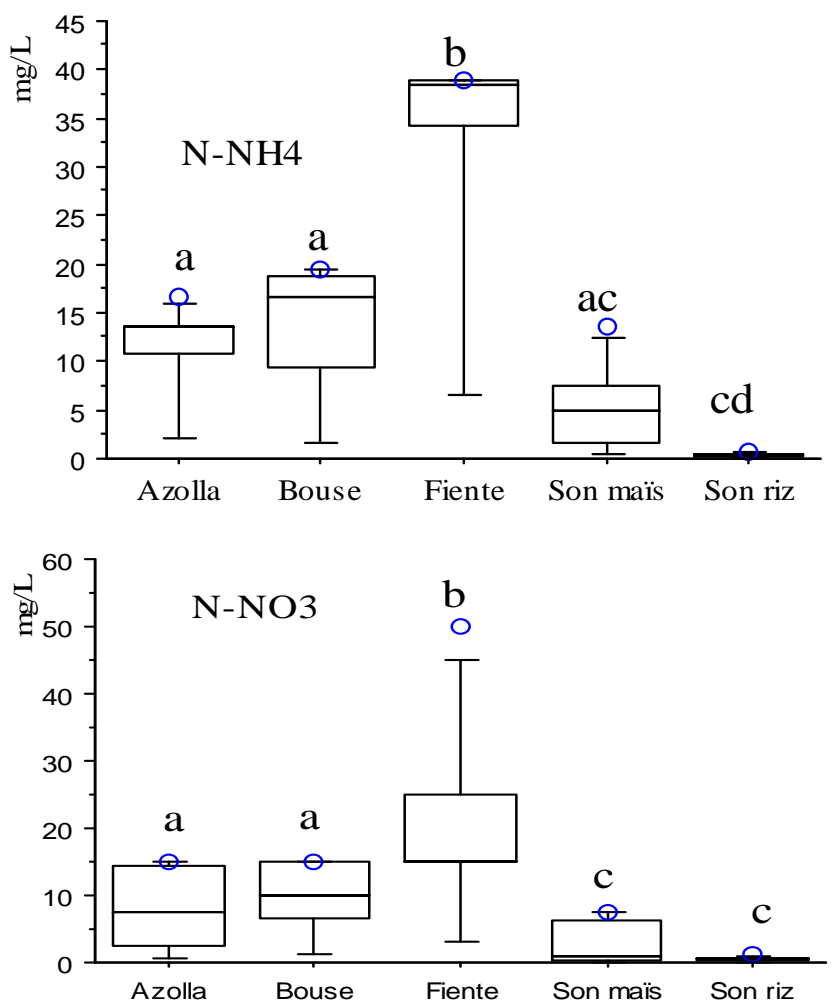

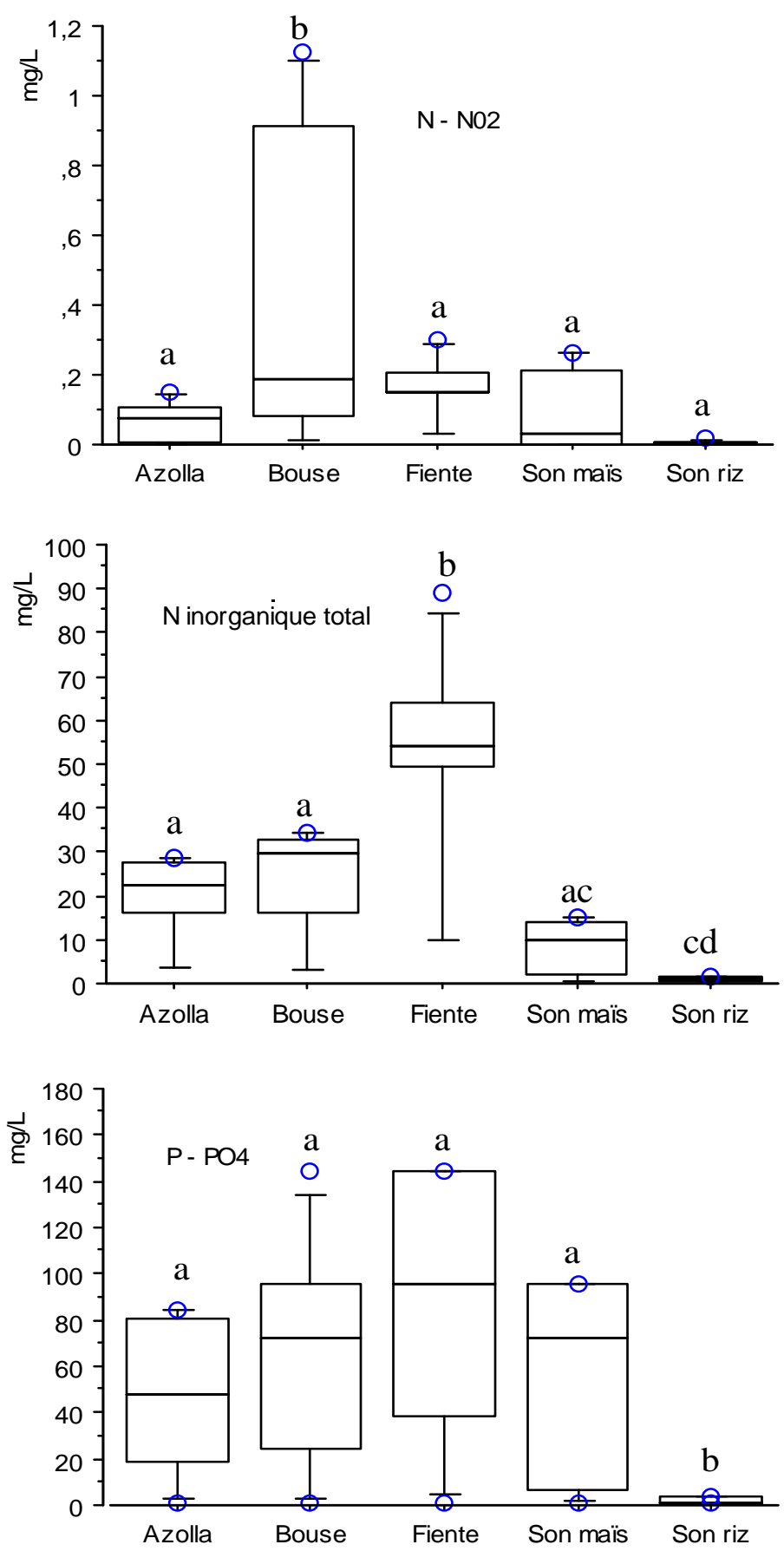

Figure 5 : Variations des sels nutritifs dans les bacs.

Pour chaque paramètre, les mêmes lettres alphabétiques sur les boîtes à moustache (box plots) indiquent qu'il $n$ 'existe aucune différence statistiquement significative $(p<0,05)$ entre les substrats. 
A
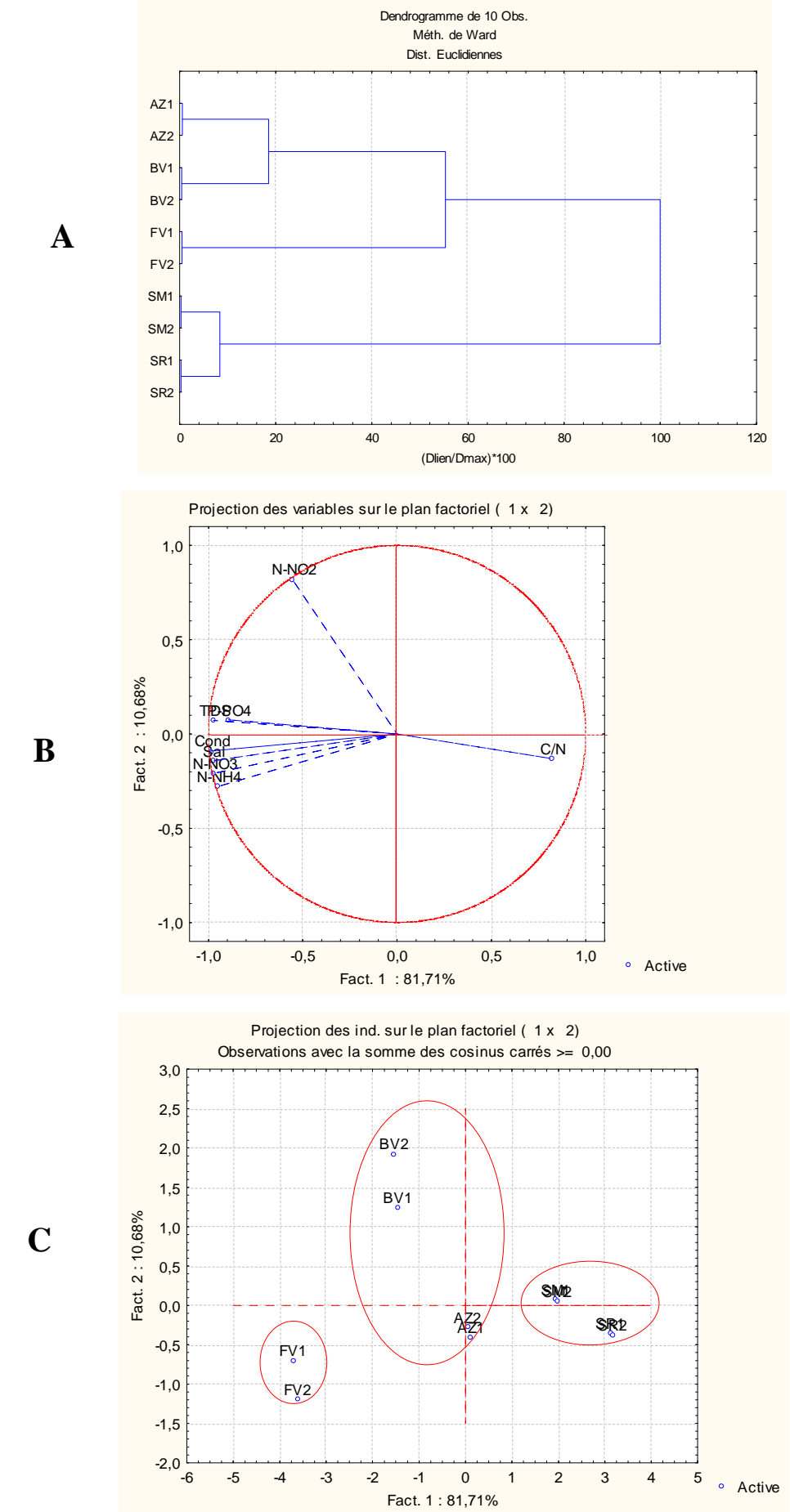

Figure 6 : Dendrogramme mettant en relief le groupement des substrats (A), analyse en composante principale mettant en relief la corrélation des variables chimiques aux axes (B) et des substrats aux axes $(\mathrm{C})$. 
Tableau 1 : Composition chimique initiale des 5 substrats.

\begin{tabular}{lccccc}
\hline Paramètres & $\begin{array}{c}\text { Azolla (Azolla } \\
\text { filiculoides) }\end{array}$ & $\begin{array}{c}\text { Bouse de } \\
\text { vache }\end{array}$ & $\begin{array}{c}\text { Fiente de } \\
\text { volaille }\end{array}$ & Son de maïs & $\begin{array}{c}\text { Son } \\
\text { de riz }\end{array}$ \\
\hline Matière sèche (\%) & 94,60 & 91,03 & 80,80 & 86,60 & 88,40 \\
Matière organique (\%) & 47,93 & 44,49 & 43,59 & 53,40 & 56,20 \\
Matière minérale (\%) & 52,07 & 55,51 & 56,41 & 46,60 & 43,80 \\
Carbone organique & 33,00 & 25,81 & 25,28 & 35,60 & 32,60 \\
total (\%) & 4,10 & 2,22 & 2,18 & 1,67 & 1,04 \\
Azote totale (\%) & 0,47 & 0,42 & 1,93 & 0,90 & 0,49 \\
Phosphate total (\%) & 4,02 & 0,39 & 1,91 & 1,19 & 0,50 \\
Potassium total (\%) & & & & & \\
\hline
\end{tabular}

\section{DISCUSSION}

L'azolla, la bouse de vache et la fiente de volaille présentent de bonnes caractéristiques chimiques de minéralisation et de libération des sels dans l'eau (Tableau 1). L'Azolla est le substrat le plus riche en azote total mais ne libère pas proportionnellement de sels azotés inorganiques dissous. Le phosphate total contenu dans ce substrat est faible et vient avant celui de la bouse de vache qui est le plus faible. La fiente de volaille présente le meilleur taux de phosphate total initial. Le taux de carbone organique total est élevé dans le son de maïs, l'azolla et le son de riz. La fiente de volaille et la bouse de vache contiennent les plus faibles taux de carbone organique total. Les résultats de la minéralisation révèlent que, la fiente de volaille libère les meilleurs taux de sels nutritifs $(\mathrm{p}<0,05)$. Elle est suivie de la bouse de vache, de l'azolla et enfin des sons. Ce résultat est conforme à celui de Knud-Hansen et al. (1991) qui ont montré que la fiente est le meilleur fertilisant organique à cause de sa solubilité plus rapide et de sa plus forte richesse en phosphore. La différence non significative entre la capacité de minéralisation et le taux de sels minéraux libérés entre l'azolla et la bouse de vache résulterait de la composition de la bouse qui ne diffère pas fondamentalement de celle de l'azolla; car la bouse de vache n'est que le reste de fourrages digérés par les bœufs. De même l'azolla à l'état sec présente un caractère très friable. Ce qui faciliterait sa décomposition comme les déjections animales. En effet, plusieurs facteurs pourraient affecter l'aptitude des substrats à la décomposition. Il s'agit notamment des caractéristiques intrinsèques de chaque substrat. Selon Heal et al. (1997), les caractéristiques intrinsèques des matières organiques sont l'un des principaux facteurs qui influencent largement leur décomposition. Ces caractéristiques propres ont été désignées sous le terme de «qualité » intrinsèque par Swift et al. (1979), qui est utilisé depuis dans les travaux relatifs à cette problématique (Cadish et Giller, 1997). Pour les substrats d'origine végétale, leur qualité chimique dépend de nombreux facteurs, notamment du type de plante (espèce), d'organe, du degré de maturité et des conditions de croissance du végétal (Sariyildiz et Anderson, 2003).

D'autres caractéristiques de la composition chimique des substrats végétaux ont été reliées à leur taux de décomposition. Des travaux ont montré que la libération du carbone sur les premiers jours de décomposition des résidus végétaux était principalement reliée à la quantité de carbone 


\section{H. AGADJIHOUEDE et al. / Int. J. Biol. Chem. Sci. 5(5): 1883-1897, 2011}

Tableau 2: Valeurs moyennes et extrêmes des paramètres relevés au cours de l'expérience.

\begin{tabular}{|c|c|c|c|c|c|c|c|c|c|c|c|}
\hline \multirow{2}{*}{ Paramètres } & & \multicolumn{2}{|c|}{$\mathbf{A Z}$} & \multicolumn{2}{|c|}{ BV } & \multicolumn{2}{|c|}{ FV } & \multicolumn{2}{|c|}{ SM } & \multicolumn{2}{|c|}{ SR } \\
\hline & & Bac 1 & Bac 2 & Bac 1 & Bac 2 & Bac 1 & Bac 2 & Bac 1 & Bac 2 & Bac 1 & Bac 2 \\
\hline \multirow{4}{*}{ TDS (mg/g) } & Moyenne & 97,32 & 99,29 & 136,38 & 138,07 & 161,67 & 159,63 & 85,23 & 87,03 & 79,75 & 78,67 \\
\hline & minimum & 30,21 & 30,21 & 30,21 & 30,21 & 30,21 & 30,21 & 30,21 & 30,21 & 30,21 & 30,21 \\
\hline & maximum & 169,8 & 173,7 & 236 & 238,8 & 224 & 222 & 140 & 141 & 130 & 128,1 \\
\hline & CV (\%) & 49,53 & 50,49 & 57,60 & 57,74 & 43,74 & 43,63 & 44,12 & 44,00 & 43,65 & 43,62 \\
\hline \multirow{4}{*}{$\begin{array}{l}\text { Conductivité } \\
(\mu \mathrm{S} / \mathrm{cm})\end{array}$} & Moyenne & 763,14 & 759,57 & 879,67 & 885,14 & 1135,13 & 1128,57 & 563,71 & 561,29 & 540,78 & 537,14 \\
\hline & minimum & 221,00 & 221,00 & 221,00 & 221,00 & 221,00 & 221,00 & 221,00 & 221,00 & 221,00 & 221,00 \\
\hline & maximum & 1210,00 & 1209,00 & 1449,22 & 1460,00 & 1675,66 & 1670,00 & 908,00 & 905,00 & 872,46 & 870,00 \\
\hline & $\mathrm{CV}(\%)$ & 47,74 & 47,82 & 53,59 & 53,78 & 45,11 & 45,14 & 42,56 & 42,39 & 42,18 & 42,32 \\
\hline \multirow{4}{*}{ Salinité (mg/L) } & Moyenne & 0,27 & 0,27 & 0,32 & 0,32 & 0,50 & 0,50 & 0,10 & 0,10 & 0,10 & 0,10 \\
\hline & minimum & 0,00 & 0,00 & 0,00 & 0,00 & 0,00 & 0,00 & 0,00 & 0,00 & 0,00 & 0,00 \\
\hline & maximum & 0,60 & 0,60 & 0,67 & 0,67 & 0,87 & 0,87 & 0,27 & 0,27 & 0,27 & 0,27 \\
\hline & CV $(\%)$ & 84,64 & 84,64 & 81,34 & 81,34 & 60,93 & 60,93 & 96,21 & 96,21 & 96,21 & 96,21 \\
\hline \multirow{4}{*}{$\mathrm{C} / \mathrm{N}$} & Moyenne & 7,84 & 7,65 & 10,7 & 11,19 & 11,36 & 11,22 & 30,39 & 32,26 & 33,97 & 35,24 \\
\hline & minimum & 7,41 & 7,37 & 8,78 & 10,12 & 10,78 & 10,67 & 26,01 & 27,06 & 28,15 & 29,12 \\
\hline & maximum & 8,25 & 7,94 & 11,61 & 11,71 & 11,59 & 11,43 & 35,36 & 37,11 & 41,37 & 41,23 \\
\hline & $\mathrm{CV}(\%)$ & 4,29 & 2,97 & 11,06 & 5,64 & 2,84 & 2,49 & 13,64 & 13,27 & 15,42 & 13,25 \\
\hline \multirow{4}{*}{ N-NH4 (mg/L) } & Moyenne & 11,99 & 11,16 & 13,81 & 12,97 & 31,80 & 32,99 & 5,44 & 5,40 & 0,36 & 0,45 \\
\hline & minimum & 0,11 & 0,11 & 0,11 & 0,11 & 0,11 & 0,11 & 0,11 & 0,11 & 0,11 & 0,11 \\
\hline & maximum & 19,50 & 13,65 & 19,50 & 19,50 & 39,00 & 39,00 & 13,65 & 13,65 & 0,55 & 0,78 \\
\hline & CV (\%) & 49,74 & 45,56 & 53,61 & 53,73 & 45,24 & 44,09 & 85,30 & 85,42 & 44,60 & 57,11 \\
\hline
\end{tabular}

1893 
H. AGADJIHOUEDE et al. / Int. J. Biol. Chem. Sci. 5(5): 1883-1897, 2011

\begin{tabular}{rlcccccccccc}
\hline & Moyenne & 7,87 & 7,87 & 10,80 & 9,37 & 20,73 & 20,73 & 2,76 & 2,76 & 0,59 & 0,59 \\
N-NO3 (mg/L) & minimum & 0,10 & 0,10 & 0,10 & 0,10 & 0,10 & 0,10 & 0,10 & 0,10 & 0,10 & 0,10 \\
& maximum & 15,00 & 15,00 & 15,00 & 15,00 & 50,00 & 50,00 & 7,50 & 7,50 & 1,10 & 1,10 \\
& CV (\%) & 80,56 & 80,56 & 55,27 & 63,95 & 74,15 & 74,15 & 121,05 & 121,05 & 61,90 & 61,90 \\
\hline Moyenne & 0,06 & 0,07 & 0,39 & 0,51 & 0,20 & 0,12 & 0,09 & 0,09 & 0,01 & 0,01 \\
N-NO2 (mg/L) & minimum & 0,00 & 0,00 & 0,00 & 0,00 & 0,00 & 0,00 & 0,00 & 0,00 & 0,00 & 0,00 \\
& maximum & 0,15 & 0,15 & 1,13 & 1,50 & 0,60 & 0,23 & 0,26 & 0,26 & 0,02 & 0,02 \\
& CV (\%) & 93,74 & 91,63 & 102,01 & 117,87 & 91,75 & 72,62 & 132,87 & 132,87 & 105,83 & 105,83 \\
\hline \multirow{2}{*}{ P-PO4 (mg/L) } & Moyenne & 46,44 & 52,61 & 69,07 & 70,10 & 92,04 & 92,04 & 54,74 & 54,74 & 1,91 & 1,91 \\
& minimum & 1,09 & 1,09 & 1,09 & 1,09 & 1,09 & 1,09 & 1,09 & 1,09 & 1,09 & 1,09 \\
& maximum & 72,00 & 96,00 & 144,00 & 144,00 & 144,00 & 144,00 & 96,00 & 96,00 & 3,60 & 3,60 \\
& CV (\%) & 63,44 & 73,20 & 72,62 & 71,88 & 65,35 & 65,35 & 82,50 & 82,50 & 67,03 & 67,03 \\
\hline
\end{tabular}


présent dans la fraction soluble à l'eau (Herman et al., 1977 ; Trinsoutrot et al., 2000; Abiven et al., 2005). La proportion de composés solubles, dégradés rapidement par les microorganismes décomposeurs a moins d'influence sur la libération du C à plus long terme (Bertrand et al., 2009). L'influence des composés présents dans la fraction insoluble (polysaccharides des parois cellulaires, lignines) sur la décomposition est quant à elle plus difficile à déterminer. Des relations entre certains de ces composés et les taux de libération du $\mathrm{C}$ et de $\mathrm{N}$ ont été mises en évidence (Heal et al., 1997 ; Trinsoutrot et al., 2000; Jensen et al., 2005). Par exemple le rapport $\mathrm{C} / \mathrm{N}$ qui est un important facteur à considérer dans le processus de minéralisation pour la fertilisation azotée (Nève et al., 2009). Selon Paul et Clark (1989), un rapport C/N faible $(\leq 25)$ favorise la minéralisation des matières organiques. Ainsi l'azolla, la fiente de volaille et la bouse de vache présentent des rapports $\mathrm{C} / \mathrm{N}$ faibles (Tableau 2) et pourraient être particulièrement intéressants en tant que fertilisants. Cela se justifie par les valeurs plus élevées des sels nutritifs libérés par ces derniers. Par contre, les sons de maïs et de riz ont un rapport $\mathrm{C} / \mathrm{N}$ élevé et seraient moins minéralisés. Ceci explique les faibles taux de sels nutritifs dissous dans les bacs à sons. La faible minéralisation des sons de riz et de maïs est due à d'autres facteurs tels que la rigidité et la taille des particules. Selon Angers et Recous (1997) et Bertrand et al. (2006), l'architecture tissulaire (rigidité de la structure pariétale, degré de lignification) et la taille des résidus végétaux sont aussi importantes que la composition chimique pour déterminer leur taux de décomposition. Alors, l'utilisation de sons de taille réduite améliorerait la dynamique de décomposition de ces substrats. Déjà, Awaïss a essayé avec succès des sons de riz micronisé dans la production du zooplancton (Awaïss et al., 1993).

Par ailleurs, la disponibilité de ces substrats doit aussi être considérée comme facteur pouvant guider dans le choix du substrat, notamment suite au développement rapide de l'aquaculture. Généralement, les déjections sont plus disponibles grâce au développement de l'élevage surtout avicole et s'obtiennent presque gratuitement. Toutefois, l'obtention de l'azolla ne présente pas trop de contrainte car il est une fougère très proliférant et très facile à produire.

\section{Conclusion}

A l'issue de cette étude, on peut retenir que :

- les forts taux de sels nutritifs sont obtenus avec les déjections animales ;

- les trois groupes de substrats se distinguent en fonction des compositions chimiques considérées traduisant leur aptitude à la minéralisation ;

- malgré que la bouse se retrouve avec l'azolla dans le groupe II, elle présente les caractéristiques plus proches de celles de la fiente de volaille.

Ainsi, les déjections animales se prêtent mieux à la fertilisation organique. Mais, un essai de production sera réalisé pour dégager avec aucun doute le meilleur substrat de production entre la bouse de vache et la fiente de volaille.

\section{REFERENCES}

Abiven S, Recous S, Reyes V, Oliver R. 2005. Mineralisation of $\mathrm{C}$ and $\mathrm{N}$ from root, stem and leaf residues in soil and role of their biochemical quality. Biol. and Fert. Soils, 42: 119-128.

Abou Y. 2007. Effet de l'alimentation à base d'Azolla sur la production du tilapia du Nil en zones humides au Bénin. Thèse de doctorat, Université Notre-Dame de la paix, Namur, p. 218.

Agadjihouèdé H, Bonou CA, Lalèyè P. 2010. Effet de la fertilisation à base des fientes de volaille sur la production du zooplancton en aquarium. Anls. Sci. Agr., 14(1): 63-75.

Ancion P-Y, Hoang TTH, Ton TP., Pham KT, Chiang CN, Dufey JE. 2009. Utilisation 
agricole de plantes aquatiques, notamment en tant qu'amendement des sols, dans la province de Thua Thien Hue, Centre Vietnam. 1. Inventaire, abondance et caractérisation chimique des plantes aquatiques disponibles localement. Tropicultura, 27(3): 144-151.

Angers DA, Recous S. 1997. Decomposition of wheat straw and rye residues as affected by particle size. Plant and Soil, 189: 197-203.

Awaïss A, Kestemont P. 1997. Dynamique de production et qualité nutritionnelle du rotifère d'eau douce Brachionus calyciflorus. Aquat. Living Resour., 10: 111-120.

Awaïss A, Kestemont P, Micha J-C. 1993. Etude du premier alevinage du poissonchat africain, Clarias gariepinus (Burchell, 1822), avec le rotifère d'eau douce, Brachionus calyciflorus (Pallas). In Production, Environnement and quality. Bordeaux Aquaculture'92. (eds by G. Barnabé \& P. Kestement). EAS Spec. Publ., 18: 443-453.

Baize D. 1988. Guide des Analyses Courantes en Pédologie. Impr. Jouve : Paris.

Bertrand I, Chabbert B, Kurek B, Recous S. 2006. Can the biochemical features and histology of wheat residues explain their decomposition in soil? Plant and Soil, 281: 291-307.

Bertrand I, Prévot M, Chabbert B. 2009. Soil decomposition of wheat internodes of different maturity stages: Relative impact of the soluble and structural fractions. Bioresource Technology doi: 10.1016/j.biortech.2008.06.019.

Bunasols. 1987. Méthodes d'analyse physique et chimique des sols, eaux et plantes. Documentation technique $\mathrm{N}^{\circ} 3$, Ouagadougou, $159 \mathrm{p}$.

Burns CW, Schallenberg M. 1998. Impacts of nutrients and zooplankton on the microbial food web of an ultraoligotrophe lake. J. Plankton Res., 20: 1501-1525.
Cadisch G, Giller KE. 1997. Driven by Nature. CAB International: Wallingford, $\mathrm{UK} ; 409$.

Dabbadie L. 1996. Etude de la viabilité d'une pisciculture rurale à faible niveau d'intrant dans le Centre-Ouest de la Côte d'Ivoire : approche du réseau trophique. Thèse de doctorat de l'Université de Paris 6 en Océanologie biologique, p. 207.

Dresboll DB, Magid J. 2006. Structural changes of plant residues during decomposition in a compost environment. Bioresource Technology, 97: 973-981.

Dillon PJ, Rigler FH. 1974. The phosphoruschlorophyll relationship in lakes. Limnol. Oceanogr., 19: 2112-2116.

Dossou S. 2008. Etude des potentialités et des contraintes de développement de la pisciculture dans la commune de Malanville au Bénin. Mémoire d'ing. Agr., 88 p.

Fenchel T. 1987. Ecology of Protozoa: the Biology of Free Living Phagotrophic Protists. Springler-Verlag; 54 p.

Hach 1991. Packaging guide. DREL/2010 Advanced Water Quality Laboratory.

Hartley HO. 1959. Smallest composite designs for quadratic response surface. Biometrics, 15: 611-624.

Heal OW, Anderson JM, Swift MJ. 1997. Plant litter quality and decomposition: an historical overview. In Driven by Nature: Plant Litter Quality and Decomposition, Cadisch G, Giller KE (Eds). CAB International: Wallingford, UK; 3-30.

Greenberg AE, Clesceri LS, Eaton AD, Franson MAH. 1992. Standard methods for the examination of water and wastewater (18th édition). Am. Publ. Health Assoc., 4: 108-1 13.

Herman WA, McGill WB, Dormaar JF. 1977. Effects of initial chemical composition on decomposition of roots of three grass species. Can. J. Soil Sci., 57: 205-215.

Jensen LS, Salo T, Palmason F, Breland TA, Henriksen TM, Stenberg B, Pedersen A, Lundstrom C, Esala M. 2005. Influence 
of biochemical quality on $\mathrm{C}$ and $\mathrm{N}$ mineralisation from a broad variety of plant materials in soil. Plant and Soil, 273: 307-326.

Jobling M, Meloy OH, Dos Santos Christiansen JS. 1994. The compensatory growth response of the Atlantic cod: effects of nutritional history. Aquacult. Int., 2(2): 75- 90.

Marty J, Pinel-Alloul B, Carrias JF. 2002. Effet de la prédation et des nutriments sur les réseaux microbiens planctoniques. Rev. Sci. Eau., 15(1): 37-49.

Knud-Hansen CF, Batterson TR, McNabb CD, Harahat IS, Sumantadinata K, Eidman HM. 1991. Nitrogen input, primary productivity and fish yield in fertilized freshwater ponds in Indonesia. Aquaculture, 94: 49 - 63.

Nève C, Ancion P-Y, Hoang Thi Thai H, Pham Khanh T, Chiang CN, Dufey JE. 2009. Fertilization capacity of aquatic plants used as soil amendment in the coastal sandy area of Central Vietnam. Comm. Soil Sci. and Plant Anal., 40 (17/18): 2658-2672.

Paul EA, Clark FE. 1989. Soil Microbiology and Biochemistery. Press Academy Inc: San Diego; 273.

Porter KG, Paerl H, Hodson R, Pace M, Priscu J, Riemann B, Scavia D, Stockner J. 1988. Microbial interactions in lake food webs. In Complex Interactions in Lake Communities, Carpentar (ed). Springer-Verlag: 209-227.

Prithwiraj J, Kripan S, Sudip B. 2004. Effect of different application rates of cowdung and poultry excreta on water quality and growth of ornamental carp, Cyprinus carpio vr. koi, in Concrete Tanks. Turk. J. Fish. Aquat. Sci., 4: 17-22.
Rigler FH, Peters RH. 1995. Science and Limnology. In Excellence, Kinne O (ed). Ecology, 6, Ecology Institute: Germany.

Robertson A. 1999. Limiting Nutrient Workshop 1997. LWRRDC, Canberra, Occasional paper 7/99, 17p.

Sariyildiz T, Anderson JM. 2003. Interactions between litter quality, decomposition and soil fertility: a laboratory study. Soil Biol. Biochem., 35: 391-399.

Sérémé A, Mey Ph, 2007. Valorisation agricole des ordures ménagères en zone soudano-sahélienne: cas de la ville de Bobo Dioulasso. Rev. CAMES-Série A, 05: 63-71.

Shappiro J. 1988. Introductory lecture at the international symposium: Phosphorus in Freshwater Ecosystems,Uppsala, Sweden, October 1985. Hydrobiologia, 170: 9-17.

Swift MJ, Heal OW, Anderson JM. 1979. Decomposition in terrestrial ecosystems. In Studies in Ecology (Vol 5). Blackwell Scientific Publications: Oxford, UK.; 372.

Ton That P, Le Van M, Le Thi Nam T. 2002. Sustainable development of aquaculture in Tam Giang lagoon,. In Lessons in Resource Management from the Tam Giang lagoon, Brzeski VJ, Newkirk GF (Eds). The Gioi Publishers: Hanoi; 27-37.

Trinsoutrot I, Recous S, Bentz B, Lineres M, Cheneby D, Nicolardot B. 2000. Biochemical quality of crop residues and carbon and nitrogen mineralization kinetics under nonlimiting nitrogen conditions. Soil Sci. Soci. America J., 64: 918-926. 